\title{
1 Sri Lankan perspective of knowledge and attitude towards genomic 2 medicine and research: protocol for a qualitative study.
}

3

4 5

6

7

8

9

Shiran Chamika W.A. ${ }^{1}$, Lydia Weiss ${ }^{2}$, Kaushalya Jayaweera ${ }^{1}$, Athula Sumathipala 1, 3 , Jonathan Ives ${ }^{4 *}$

${ }^{1}$ Institute for Research \& Development, Colombo, Sri Lanka.

${ }^{2}$ College of Medical and Dental Sciences, University of Birmingham, Birmingham, UK

${ }^{3}$ Research Institute for Primary Care \& Health Sciences, Faculty of Medicine \& Health Sciences, Keele University, UK

${ }^{4}$ Centre for Ethics in Medicine, Bristol Medical School, University of Bristol, UK

Shiran Chamika W.A. - shiranchamika@gmail.com

Lydia Weiss - LLW287@student.bham.ac.uk

Kaushalya Jayaweera - kaushj@gmail.com

Athula Sumathipala - a.sumathipala@keele.ac.uk

Jonathan Ives- j.ives@bristol.ac.uk

Abstract

\section{Background}

Genomic research allows new discoveries to alleviate human suffering but in turn raises significant ethical issues. In this context, public acceptance and engagement is a major determinant of optimization of genomic research. Therefore, understanding public perspectives related to genomic research is imperative, as it provides a foundation to address ethical issues which may be unknown to researchers and other stakeholders. Although genomic medicine and research has developed in Sri Lanka during the past decade, there is a paucity of understanding about public knowledge and attitudes towards it.

\section{Methods}

This research, therefore, aims to explore the understanding, knowledge and attitudes of a Sri Lankan public towards genomic medicine and research, in order to consider more effective and 
appropriate ways of communicating about the risks and benefits of participating in genomic research. Twenty four participants will be purposively selected from a previously conducted Colombo Twin and Singleton follow-up study (COTASS-2). In-depth qualitative interviews with a semi structured topic guide will be used to collect data, which will be analysed using conventional content analysis.

\section{Discussion}

This study is the first of its kind to explore public perspectives towards genomic medicine and research in Sri Lanka. Findings may have implications for producing guidelines for ethical genomic research in Sri Lanka.

\section{Key words}

Genomic medicine, genomic research, Ethics, Sri Lanka

\section{Background}

Genomic medicine has, arguably, now entered the medical mainstream, and is fast becoming a critical component in medical practice and research with the expectation that it can provide powerful tools to alleviate human suffering [1]. Interest in, and engagement with, genomic medicine and research has consequently increased globally during the past few decades at a rapid rate [2] with numerous technological advances and new discoveries about the genetic basis of many diseases [3].

Obtaining genuine informed consent for participation in research is challenging, and particularly so for research in a field that is fast developing. Traditional approaches to designing informed consent processes rely on 'experts' determining what information participants need to know in order to make an appropriately informed decision, but this model is increasingly viewed as problematic for its failure to be attentive to the needs, values and beliefs of the communities engaged by the research [4]. Additionally, and from a more 
pragmatic perspective, since community acceptance and engagement is a major factor in the success of genomic research $[5,6]$, more attention to community engagement is required in order to develop appropriate informed consent processes [7].

Participants' literacy, knowledge, and attitudes toward genomic research play a vital role in this context[2,8-11], and these are likely to vary depending on geographical, cultural, and social differences $[9,12-14]$. Consequently, it cannot be assumed that informed consent practices developed in one context will be appropriate for all. Additionally, given that genomic screening and diagnostic technologies can potentially obtain information about an individual that also provides information about disease and disease risk pertaining to family members or specific populations (including, for example, tribal communities [15]), it is important that genomic research planning directly involves the populations under study [16], and not just the individuals.

There is, therefore a need to understand the culture, values, beliefs, norms and attitudes of the specific research population with whom we are seeking to engage, prior to introducing genomic research. It is only through this engagement that appropriately contextualised informed consent processes can be developed.

Although Sri Lanka is a developing country, genomic developments from other parts of the world are reaching its shores increasingly rapidly, due to the advancement of information technology [17]. For example, genetic and molecular diagnostics were introduced in Sri Lanka around 2001, and local genomic research and development competencies have grown progressively since then [16].

The importance of building capacity for research and development of genomic medicine has been recognised in Sri Lanka, where a basic infrastructure and institutional support already exists [16]. At present, there are several state-owned and private institutions engaging in 
molecular diagnostics and genomic research, on a variety of scales and with different aims and perspectives. For example, the Institute for Research and Development (IRD) is a nongovernmental, not-for-profit institution that has established a genetic laboratory and bio-bank as part of their Colombo Twin and Singleton follow up Study (COTASS-2)[18,19]. This biobank will be used to undertake future epigenetic, genome wide association, and candidate gene studies in order to better understand the gene - environment interplay in several medical conditions [18]. However, recruitment to this bio-bank has seen mixed success, raising several questions by the investigators about the extent to which potential participants sufficiently understand the potential benefits of such future research, but also how this understanding can be developed in a balanced and ethical manner. [20].

There is indirect evidence of negative attitudes toward genetic research in Sri Lanka [18]. A previous IRD study has highlighted potential limitations in the public's understanding of 'research' by exploring the views of the public and professionals [21]. Some information about genomic research and medicine in Sri Lanka comes from media reports, which tend to be sensationalised and highlight the exploitation of Sri Lankan genetic heritage through the exportation of genetic material abroad [18]. Such reports may generate negatives attitudes within communities towards genetics research. Additionally, the IRD met substantial resistance when founding the genetics laboratory, taking five years for it to be established [22]. A limited number of studies [23-26] have found a positive attitude towards genomics amongst health professionals in Sri Lanka. These data, however, represent the views of a minority expert group rather than those of the public, whose engagement is likely to be key to its success. Even with the support of medical professionals, the potential for the success of genomic medicine cannot be explored without public participation. There remains a need for a more nuanced understanding of what research participants and their communities understand by medical research in general and genetic research in particular [27], so that information can be tailored 
and communicated in a way that achieves the goals of obtaining both informed consent and informed refusal.

This research, therefore, aims to explore the understanding, knowledge and attitudes of the Sri Lankan public towards genomic medicine and research, in order to develop more effective and appropriate ways of communicating about the risks and benefits of participating in genomic research.

\section{Method}

\section{Research questions}

To meet the aim stated above, this project seeks to answer three key questions:

1. What do people in Colombo, Sri Lanka, understand about the aims and purpose of genomic medicine and research?

2. What do they think are the risks and benefits of genomic medicine and research?

3. Do they think that genomic medicine and research should be promoted and/or supported, and why?

\section{Sampling}

All participants will be sampled from the IRD, Colombo Twin and Singleton Follow-up Study (COTASS-2) participant lists[19]. As such, all potential participants will have been involved in a study collecting bio-specimens, and will have been asked if their material can be stored for bio-banking and future genetics research. Participants will include a mix of those who have consented to their genetic material being used in a bio-bank, and those who have refused consent. We can therefore expect all potential participants to have some understanding and experience of the topic. Whilst this places limitations on the study insofar as the knowledge and understandings of participants may not exactly reflect those of other members of the 
general public, it is justified because it aids the sampling process and means that participants will more likely be able to talk about the subject. This approach allows us select a particular subset of the public who have prior experience of participating in such research, as opposed to having to consider it in a hypothetical manner. This seems to be an appropriate starting point for this research, which will go on to explore attitudes amongst the wider public in later iterations.

All participants of the COTASS-2 study were invited to donate to the IRD bio-bank and out of the 5899 participants, 3483 gave consent for this. Purposive sampling will be employed to achieve a range of ages and an equal gender split. The aim will be to gain a diversity of perspectives, rather than to achieve generalizability. There will also be an equal split between those who did consent to bio-banking and those who did not, in order to achieve a better understanding of why members of the public do or do not wish to donate to bio-banks.

Due to the large volume of data that will be collected per interview, and the limited time available to collect the data, a maximum of 24 interviews will be undertaken. This number is appropriate for qualitative studies where the purpose of the research is to collect rich data and acquire an insight into the knowledge and attitudes of study participants [28]. In the event that data saturation is reached earlier than that, interviewing will stop.

\section{Inclusion and exclusion criteria}

Participants will be included in the study if (i) they are participants in the COTASS-2 Study;

(ii) live in the district of Colombo, Sri Lanka (to make travelling to an interview possible); (iii) and are willing to take part in the study. Participants will be excluded from the study only if they are unable to give consent. Participants who are illiterate will not be excluded, and measures will be taken to ensure that illiterate persons are appropriately consented. Ethical 
approval was granted for this project by the Ethics Review Committee of the Sri Lanka Medical Association, Sri Lanka (no: ERC/16 - 018).

\section{Recruitment}

Recruitment of participants will be via the IRD, based in the Colombo district of Sri Lanka.

The contact details of COTASS-2 participants are held by the IRD. All COTASS-2 participants are either members of the Sri Lankan Twin Registry (SLTR), or singletons who are part of the COTASS cohort, and all participants are from various socio-economic backgrounds. Initially, a sample of 50 potential participants will be selected to be approached, stratified by age (equal split of 18-29, 30-49, 50+), gender and involvement with the bio-bank (equal split of those who consented to donate to the bio-bank and those who did not). Selected potential participants will be approached by IRD staff via a telephone call using the contact details already recorded. At the time of this call, they will be told basic information about the study and asked if they would like to receive more information. If they would like more information, this can either be delivered verbally over the phone or posted out to them (depending on preference).

If potential participants wish to receive more information by post, they will be sent a recruitment package comprising of a letter of invitation, and participant information sheet. If the potential participant wishes to receive more information over the phone, the letter of invitation and the participant information sheet will be read out to them, however at this point paper copies will also be sent to them in the post.

If more information has been requested a follow up call will be arranged at a mutually convenient time, and the potential participants can confirm whether or not they would like to take part. This follow up call will be made by an IRD staff member. convenient time and setting will be arranged for the interview to take place. 
177 Immediately prior to the interview taking place, signed consent will be obtained, witnessed and 178 countersigned by the researcher. During the consent process, participants will be given further opportunity to ask questions about the project, and key information will be repeated.

180 Participants will be advised that they can withdraw from the study before or during the interview and up to one week after their interview date, and they will be told this in the participant information sheet and on the consent form. Data cannot be withdrawn from the study after that date, but participants may ask for their quotes not to be included in the writeup. Again, they will be told about this in the participant information sheet and consent form.

Participants will be offered 500LKR as compensation for their time at the end of the interview, which is viewed as appropriate in the local setting. If a participant chooses to withdraw from the study or suspend the interview, they will still receive the 500LKR.

The contact for the participants throughout the duration of the study will be an IRD staff member and co-investigator, who will liaise with the IRD researcher carrying out interviews.

\section{Data collection}

191 Data will be collected by a trained qualitative researcher employed by the IRD, using face-toface semi-structured qualitative interviews, comprising open-ended questions. The semistructured style of interview will allow for key themes to be covered but will not constrain or limit the participants' opportunity to express their attitudes and beliefs [29]. Face-to-face interviews will allow for a personal rapport to be built which may ease the discussion of more sensitive areas [29]. Interviews will last for around one hour. An interview may be stopped at any point by the participant, or extended by mutual agreement between the interviewer and participant. 
The interviews will be audio-recorded on an electronic recording device, to allow the detail of the interview to be accurately documented. The recording will be transferred to a secure encrypted computer and encrypted USB storage device at the earliest opportunity.

Interviews will take place at a mutually convenient time, and a mutually convenient place. This may be in the participant's own home, or at the IRD. Prior to booking an interview at the participant's home or IRD, it will be ensured that there will be a private space available for the interview to take place in.

\section{Data management and storage}

207

208

The participants' names will be recorded and each will be linked to a participant identification number via a key, which will be required in case of participants' withdrawal. The key will be stored on a secure password protected computer, in an encrypted file, in a separate folder, and on paper in a locked filing cabinet in the IRD's secured data room. Only AS, KJ, JI and the researcher will have access to the key.

Consent forms, which cannot be anonymised, will be kept in a separate location to the research data. Hard copies of the consent forms will be stored in a locked filling cabinet in the IRD's secured data room, and scanned images of them will be backed up on a secure electronic password encrypted computer.

All of the electronic field data, including all audio-recordings of interviews, will be stored securely to ensure participant confidentiality, and linked to identification numbers only. They will be stored on a password encrypted computer and backed-up onto an encrypted USB drive. Electronic transcripts will be stored in the same way. Following the transfer of the audiorecordings to the encrypted devices, they will be deleted from the recording device. Hard copies of anonymised transcripts (which will be printed out to aid initial analysis) will be stored securely in a locked filing cabinet on IRD premises. 
Digital anonymised and encrypted transcripts of interviews may be sent by secure email between members of the research team in order to conduct analysis checking prior to face to face analysis meetings. After the conclusion of the study, copies of all electronic data, hard copy transcripts and consent forms will be stored at the IRD, in the custody of AS, in accordance with IRD regulations.

\section{Data analysis}

Data analysis will be carried out by the researcher with support from AS, KJ and JI. All interview data will be transcribed verbatim and translated into English (to allow JI to contribute to analysis) and a method of conventional content analysis will be used, where codes and themes are developed directly from the text [30]. Conventional content analysis is appropriate for this study design as the aim of the research is to gain insight into the topic being studied and there is minimal existing research into the area [29]. The aim will be for codes and categories to emerge from the data, rather than to impose pre-conceived theories or ideas onto it [30]. Analysis will commence with the interview transcripts being read thoroughly to allow immersion in the data, initial first impressions will be documented at this point [28]. Following this, the data will be read again in greater detail [30]. Words in the text that appear to have significance and encompass key ideas or views, will be highlighted and coded [30]. These codes will be grouped into categories depending on links between them and a hierarchy will be created whereby smaller sub-categories are grouped into bigger categories, each representing a thematic unit [30]. Links will then be explored between these thematic units [30]. To improve the credibility of the results there will be analytic triangulation between all researchers, where the preliminary codes will be reviewed, discussed and agreed upon [28].

\section{Discussion}

\section{Benefits}


247 Benefits of this research are several-fold. Potential beneficiaries include researchers, who will

248 benefit from an enhanced understanding of possible public perspectives about genomic

249

250

251

252

253

254

255

256

257

258

259

260

261

262

263

264

265

266

267

268

269

research and genomic medicine. Specifically, and immediately, it will help the IRD work harmoniously with the local community in developing genomic research. Public engagement and support is seen as crucial to the success of genomics [6], and using the results of the study, the IRD will be better able to develop effective and appropriate communication strategies and consenting processes. Accordingly, the IRD will have greater potential to reach their goal of establishing and increasing the capacity for genomics in the country. The public will consequently potentially benefit from better understanding of genomic medicine and research, and ultimately reaping the benefits of carefully and appropriately conducted genomic research.

\section{Dissemination}

The results will be submitted for publication, which we will try to ensure is in an open access international journal, in order to make them accessible to members of the community, and the publication will be jointly authored by the Sri Lankan and UK collaborators.

A research summary will be written, and the IRD will pass it on to relevant authorities and interested parties as appropriate. The participants of the study will be able to access the online publication if they have internet access, but all participants will also be asked in their consent form whether they would like to be sent the research summary, which will be translated into local languages as appropriate. It will then be the responsibility of the IRD to pass on the research summary to participants if requested.

\section{Ethical issues}

Consent is an important issue to consider, particularly as this project involves contacting people who have already participated in research and therefore may feel they have a prior relationship 
with the recruiting site. Everything possible will be done to ensure that potential participants do not feel pressured into taking part.

Confidentiality is also an important issue. If interviews are conducted at a participant's home, it may be that participants do not wish to share their attitudes and understandings of genomics in front of family members during interviews. Where possible a private space will be found within the home for the interview to take place. Alternatively, all participants have the option of being interview at the IRD in a quiet and private room. The recruiters will all understand fully the requirements for confidentiality. All data will be stored securely, and all data will be published anonymously.

Whilst it is impossible to predict all topics that will be covered in a qualitative interview, it is possible that the interviews could bring up potentially sensitive topics about, for example, familial disease or issues about personal religious belief. Participants will be informed that they are able to stop the interview at any time, and can withdraw from the research during the withdrawal period. They will also be reminded that taking part is voluntary and that there is a guarantee of confidentiality and data anonymity. If a participant does get upset during the interview, or it becomes apparent that they need support or more information about personal issues, they will be referred, with their permission, to a member of the IRD who is qualified to provide advice and information as needed.

There is a small_risk that interviews might bring up information that suggests a participant has been misinformed about, or misunderstands, the nature of their previous research participation with the IRD. This may create a potential conflict of interest if it is critical of the IRD. Any such case, with the affected participant's permission, will be reported to Ethics Review Committee of the Sri Lanka Medical Association.

\section{List of abbreviations:}




\section{Declarations}

297

Ethics approval: Ethical approval was granted for this project by the Ethics Review Committee of the Sri Lanka Medical Association, Sri Lanka (no: ERC/16 - 018).

Consent for publication: Not Applicable

Availability of data and material: Not Applicable

Competing interests: The authors declare that they have no competing interests.

Funding: This study was supported by the Wellcome Trust (Grant reference number: 204106/Z/16/Z)

Authors' contributions: SC and LW equally contributed. LW wrote the protocol and SC wrote the manuscript. JI, AS, KJ revised the protocol and manuscript, provided critical inputs. All authors read and approved the final manuscript.

Acknowledgements: Not Applicable

Authors' information (optional):

\section{References}

1. Collins FS, McKusick VA. Implications of the Human Genome Project for Medical Science. JAMA. 2001;285:540. doi: 10.1001/jama.285.5.540

2. Wonkam A, Njamnshi AK, Angwafo FF. Knowledge and attitudes concerning medical genetics amongst physicians and medical students in Cameroon (sub-Saharan Africa). Genet. Med. 2006;8:331-8. doi:10.1097/01.gim.0000223542.97262.21

3. Bloss CS, Jeste D V, Schork NJ. Genomics for Disease Treatment and Prevention. Psychiatr Clin North Am. 2011;34:147-66. doi: 10.1016/j.psc.2010.11.005 
4. Ives J, Damery S, Redwod S. PPI, paradoxes and Plato: who's sailing the ship? J. Med. Ethics. Institute of Medical Ethics; 2013;39:181-5. doi: 10.1136/medethics-2011-100150

5. Calsbeek H, Morren M, Bensing J, Rijken M. Knowledge and Attitudes Towards Genetic Testing: A Two Year Follow-Up Study in Patients with Asthma, Diabetes Mellitus and Cardiovascular Disease. J. Genet. Couns. 2007;16:493-504. doi:10.1007/s10897-006-9085-9

6. Etchegary H, Green J, Parfrey P, Street C, Pullman D. Community engagement with genetics: Public perceptions and expectations about genetics research. Heal. Expect. 2015;18:1413-25. doi:10.1111/hex.12122

7. Upshur RE, Lavery J V, Tindana PO. Taking tissue seriously means taking communities seriously. BMC Med. Ethics. 2007;8. doi:10.1186/1472-6939-8-11

8. Condit CM. Public understandings of genetics and health. Clin. Genet. 2010;77:1-9. doi:10.1111/j.1399-0004.2009.01316.x

9. Haga SB, Barry WT, Mills R, Ginsburg GS, Svetkey L, Sullivan J, et al. Public knowledge of and attitudes toward genetics and genetic testing. Genet. Test. Mol. Biomarkers. 2013; 17:327-35. doi: 10.1089/gtmb.2012.0350

10. Jamal L, Sapp JC, Lewis K, Yanes T, Facio FM, Biesecker LG, et al. Research participants ' attitudes towards the confidentiality of genomic sequence information. Eur. J. Hum. Genet. Nature Publishing Group; 2014;22:964-8. doi:10.1038/ejhg.2013.276

11. Shabani M, Bezuidenhout L, Borry P. Attitudes of research participants and the general public towards genomic data sharing: a systematic literature review. Expert Rev. Mol. Diagn. 2014;14:1053-65. doi:10.1586/14737159.2014.961917.

12. Aro A., Hakonen A, Hietala M, Lönnqvist J, Niemelä P, Peltonen L, et al. Acceptance of genetic testing in a general population: age, education and gender differences. Patient Educ. Couns.1997;32:41-9. doi: 10.1016/S0738-3991(97)00061-X

13. Catz DS, Green NS, Tobin JN, Lloyd-Puryear MA, Kyler P, Umemoto A, et al. Attitudes about Genetics in Underserved, Culturally Diverse Populations. Public Health Genomics. 2005;8:161-72. doi: 10.1159/000086759

14. de Vries J, Bull SJ, Doumbo O, Ibrahim M, Mercereau-Puijalon O, Kwiatkowski D, et al. Ethical issues in human genomics research in developing countries. BMC Med. Ethics.; 2011;12:5. doi: 10.1186/1472-6939-12-5

15. Adlan AAA. The biomedical ethics of donating blood for molecular and genetics research in Saudi Arabia. University of Birmingham; 2015. http://etheses.bham.ac.uk/6085/. Accessed 12 Dec 2017.

16. Manamperi A, Huzair F. Capacity Building in Genomics Medicine and Molecular Diagnostics: The Case of Sri Lanka. Curr. Pharmacogenomics Person. Med. 2012;10:185-94. doi: $10.2174 / 187569212802509960$

17. Sirisena ND, Neththikumara N, Wetthasinghe K, Dissanayake VHW. Implementation of genomic medicine in Sri Lanka: Initial experience and challenges. Appl. Transl. Genomics. Elsevier B.V.; 2016;9:33-6. doi:10.1016/j.atg.2016.05.003

18. Sumathipala A, Siribaddana S, Hotopf M, McGuffin P, Glozier N, Ball H, et al. The Sri Lankan Twin Registry: 2012 Update. Twin Res. Hum. Genet. 2013;16:307-12. doi:10.1017/thg.2012.119 
19. Jayaweera K, Aschan L, Pannala G, Adikari A, Glozier N, Ismail K, et al. The Colombo Twin and Singleton Follow-up Study: a population based twin study of psychiatric disorders and metabolic syndrome in Sri Lanka. BMC Public Health. 2018;18:145. doi:10.1186/s12889-017-4992-2

20. Haga SB, Beskow LM. Ethical, Legal, and Social Implications of Biobanks for Genetics Research. Adv. Genet. 2008. p. 505-44. doi: 10.1016/S0065-2660(07)00418-X

21. Sumathipala A, Siribaddana S, Hewage S, Lekamwattage M, Athukorale M, Siriwardhana C, et al. Understanding of research: a Sri Lankan perspective. BMC Med. Ethics. 2010;11. doi: 10.1186/1472-6939-11-7

22. Siriwardhana C, Sumathipala A, Siribaddana S, Samaraweera S, Abeysinghe N, Prince $\mathrm{M}$, et al. Reducing the scarcity in mental health research from low and middle income countries: A success story from Sri Lanka. Int. Rev. Psychiatry. 2011;23:77-83. doi:10.3109/09540261.2010.545991

23. Dissanayake VHW, Simpson R, Jayasekara RW. Attitudes towards the new genetic and assisted reproductive technologies in Sri Lanka: A preliminary report. New Genet. Soc. 2002; 21:65-74. doi: 10.1080/14636770220122773

24. Simpson B. Ethical regulation and the new reproductive technologies in Sri Lanka: perspectives of ethics committee members. Ceylon Med. J.2014;46:54. doi:10.4038/cmj.v46i2.6493

25. Jayasekara R, Kristl GB, Wertelecki W. Acceptance of genetic services: a study of physicians in Colombo, Sri Lanka. J. Biosoc. Sci.1988;20:1-7. doi:10.1017/S0021932000017193

26. Jayasekara RW. The attitudes of doctors and students towards a genetic service in an Asian country: Sri Lanka. Asia. Oceania. J. Obstet. Gynaecol.1989;15:267-70. http://www.ncbi.nlm.nih.gov/pubmed/2597090

27. Kengne-Ouafo JA, Millard JD, Nji TM, Tantoh WF, Nyoh DN, Tendongfor N, et al. Understanding of research, genetics and genetic research in a rapid ethical assessment in north west Cameroon. Int. Health. 2016;8:197-203. doi:10.1093/inthealth/ihv034

28. Braun V, Clarke V. Successful Qualitative Research: A Practical Guide For Beginners. Success. Qual. Res. A Pract. Guid. Beginners. London, SAGE; 2013. http://eprints.uwe.ac.uk/21156

29. Ives J, Damery S. Qualitative Methodology. In: Dawn-Marie Walker, editor. An Introd. to Heal. Serv. Res. A Pract. Guid.SAGE; 2014. p. 99-115.

30. Hsieh H-F, Shannon SE. Three Approaches to Qualitative Content Analysis. Qual. Health Res. 2005;15:1277-88. doi:10.1177/1049732305276687 